\title{
Respuesta bioquímica y morfométrica a la incorporación de pescado, pollo y carne vacuna en la dieta de Caiman yacare en cautiverio
}

\author{
Koza, G.A.; Mussart, N.B.; Barboza, N.N.; Coppo, J.A. \\ Cátedra de Fisiología, Facultad de Ciencias Veterinarias, UNNE, Sargento Cabral 2139, Corrientes (3400), \\ Argentina. Tel/Fax:03783-425753 (int 153). E-mail: fisiologia@vet.unne.edu.ar.
}

\begin{abstract}
Resumen
Koza, G.A.; Mussart, N.B.; Barboza, N.N.; Coppo, J.A.: Respuesta bioquímica y morfométrica por incorporación de pescado, pollo y carne vacuna a la dieta de Caiman yacare en cautiverio. Rev. vet. 23: 2, 110-115, 2011. Los indicadores bioquímicos nutricionales, el peso y las dimensiones corporales, permiten evaluar el impacto de distintas dietas sobre el crecimiento de Caiman yacare, así como optimizar su cría comercial. En un establecimiento del nordeste argentino se evaluaron tres dietas utilizando 60 ejemplares sub-adultos de dicha especie (peso: 1,46 $\pm 0,06 \mathrm{~kg}$ y longitud hocico-cloaca: $37,29 \pm 0,28 \mathrm{~cm}$ ), divididos en tres lotes de 20 animales cada uno (50\% de cada sexo). Todos recibieron una dieta base (70\%) de alimento balanceado (MS 88\%, PB 30\%, EE $11 \%$, cenizas 4,5\%) y un suplemento (30\%) que varió según el grupo (carne vacuna, pollo o pescado). Al inicio y al final de la experiencia (170 días) se realizaron pesajes y mediciones corporales de los reptiles. Además, se efectuaron análisis sanguíneos (perfil nutricional) a 10 animales de cada lote (50\% de cada sexo). A través de un diseño completamente aleatorizado se realizó el análisis de la covarianza (ANCOVA). Las variables longitud y ancho de cabeza, así como los valores de proteínas totales, albúmina, relación albúmina-globulina, hematocrito, hemoglobina, glucosa y potasio no revelaron diferencias significativas entre dietas. Los caimanes que recibieron la ración suplementada con pollo, mostraron mayores incrementos de peso vivo, longitud total, longitud hocico-cloaca y perímetro torácico, así como niveles superiores de globulinas totales, globulina beta, colesterol total, C-LDL, sodio y fósforo inorgánico $(\mathrm{p}<0,05)$. Los animales que consumieron pescado exhibieron niveles sanguíneos significativamente mayores de alfa y gamma globulinas, creatinina, C-HDL, fructosamina y calcio. Los reptiles suplementados con carne vacuna presentaron mayores niveles de urea, triglicéridos y magnesio $(p<0,05)$. En ningún caso se registraron cuadros compatibles con deficiencias nutricionales. Por el contrario, los resultados indican que los tres suplementos utilizados revelaron ser útiles para la alimentación de C. yacare, especialmente el de origen aviar por su significativa repercusión sobre los aumentos de peso y dimensiones corporales de los caimanes.
\end{abstract}

Palabras clave: Caiman yacare, nutrición, desarrollo, valores bioquímicos, dimensiones corporales.

\begin{abstract}
Koza, G.A.; Mussart, N.B.; Barboza, N.N.; Coppo, J.A.: Biochemical and morphometrical response to the supplementation of fish, chicken and bovine meat in the diet of Cayman yacare captive specimens. Rev. vet. 23: 2, 110-115, 2011. Nutritional biochemical indicators, liveweight and corporal dimensions allow to evaluate the impact of different diets regarding the growth of Caiman yacare, as well as to optimize their commercial breeding. In a hatchery located in northeast Argentina, three diets were evaluated using 60 sub-adults specimens (liveweigh: $1.46 \pm 0.06 \mathrm{~kg}$ and muzzle-sewer longitude: $37.29 \pm 0.28 \mathrm{~cm}$ ), divided in three groups of 20 animals each (50\% each sex). All animals received a basal diet (70\%) of balanced food (DM $88 \%$, CP $30 \%$, EE $11 \%$, ash $4.5 \%$ ) and a supplementation (30\%) that varied according to the group (bovine meat, chicken or fish). At the beginning and the end of the trial (170 days) weighings and corporal measurements of the reptiles were carried out. Also, blood analysis (nutritional profile) were performed in 10 animals of each group (50\% of each sex). Through a totally randomized design, the analysis of the covariance (ANCOVA) was carried out. Parameters such as longitude and head wide, as well as values of total pro-
\end{abstract}


tein, albumin, albumin-globulin ratio, hematocrit, hemoglobin, glucose and potassium didn't reveal significant differences among diets. Caimans that received the food supplemented with chicken showed higher increments of liveweight, total longitude, muzzle-sewer longitude, and thoracic perimeter, as well as superior levels of total globulin, beta globulin, total cholesterol, C-LDL, sodium and inorganic phosporous $(\mathrm{p}<0.05)$. Animals that received fish exhibited significantly higher blood levels of alpha and gamma globulins, creatinine, C-HDL, fructosamine and calcium. Reptilian supplemented with bovine meat presented higher levels of urea, triglycerides and magnesium $(\mathrm{p}<0.05)$. No cases compatible with nutritional deficiencies were registered. On the contrary, results indicate that the three supplements revealed to be useful for feeding C. yacare, especially with chicken meat for its significant repercussion on the increment of liveweight and corporal dimensions of caimans.

Key words: Caiman yacare, nutrition, growth, biochemical values, corporal dimensions.

\section{INTRODUCCIÓN}

En el nordeste argentino habitan los crocodílidos denominados yacaré overo (Caiman latirostris) y yacaré negro (Caiman yacare) ${ }^{4}$. Su distribución natural abarca las cuencas de los ríos Paraná, Paraguay, São Francisco y Uruguay ${ }^{3}$. La cría racional de los caimanes autóctonos en condiciones de cautiverio ha evolucionado a lo largo del tiempo, con el fin de alcanzar rápidamente una mejor calidad de cuero y carne, en relación al producto obtenido de los animales capturados de la naturaleza ${ }^{15}$.

En los últimos años su comercialización motivó la proliferación de criaderos donde se practica el sistema ranching, consistente en la recolección de huevos del medio natural, con eclosión y ulterior crianza de los caimanes en condiciones controladas, hasta que alcancen el tamaño adecuado para su sacrificio y venta ${ }^{17,23}$. Este sistema facilita el conocimiento de la situación de las poblaciones silvestres a partir del trabajo permanente en las áreas de nidificación, y por otra parte refirma el compromiso de conservación y control de la fauna autóctona pues promueve la reintroducción de los caimanes obtenidos mediante reproducción y cría en cautividad ${ }^{8}$. Este diseño tiene como fin la comercialización de la generación F2, así como la repoblación de la zona con los yacarés en edad de sobrevida asegurada ${ }^{22}$. La implementación de los programas de manejo sustentable de caimanes se practica con éxito en Argentina desde hace varios años ${ }^{7}$.

En los criaderos, la eficacia de las dietas implementadas puede comprobarse mediante los aumentos del peso y dimensiones corporales, lo cual debería traducirse en un incremento en la producción de carne y cuero, principales propósitos de la cría en cautiverio ${ }^{14}$. Se admite que el costo de la alimentación de los caimanes en cautiverio representa del 50 al $60 \%$ del valor total de la producción ${ }^{18}$.

Para mejorar la alimentación y, por ende, el estado de salud de estos reptiles, actualmente se investigan parámetros fisiológicos que operan como indicadores nutricionales y metabólicos, los cuales aunados a la evolución del peso y dimensiones corporales permiten optimizar la rentabilidad de la producción ${ }^{1}$.

El objetivo del presente trabajo fue comparar los efectos de tres dietas diferentes, sobre el desarrollo (peso vivo, tamaño corporal) y medio interno (perfiles bioquímicos sanguíneos) de ejemplares de C. yacare en crecimiento, en un criadero del nordeste argentino.

\section{MATERIAL Y MÉTODOS}

La experiencia se realizó en un establecimiento de la localidad de Santa Ana (Corrientes, Argentina). Se emplearon 60 ejemplares sub-adultos de C. yacare clínicamente sanos $(50 \%$ de cada sexo). En promedio, el peso vivo (PV) inicial fue de 1,46 $\pm 0,06 \mathrm{~kg}$ y la longitud hocico-cloaca ( $\mathrm{LH})$ de $37,29 \pm 0,28 \mathrm{~cm}$. Los sujetos experimentales se dividieron en tres lotes de 20 animales cada uno ( $50 \%$ de cada sexo), los cuales permanecieron alojados en piletas separadas.

La dieta base consistió en un alimento balanceado (MS $88 \%$, PB 30\%, EE $11 \%$, cenizas 4,5\%) y fue la misma para todos los grupos (70\% de la ración). El $30 \%$ restante estuvo constituido por carne vacuna (dieta A), trozos de pollo (dieta B) y pescado de río (dieta C). Los caimanes de los tres grupos fueron alimentados ad libitum de lunes a viernes, durante 170 días, con una oferta equivalente al $25 \%$ del PV promedio.

$\mathrm{Al}$ inicio y al final de la experiencia se realizaron pesajes (balanza) y mediciones corporales (cinta métrica metálica) a todos los sujetos experimentales, que incluyeron parámetros como longitud total (LT), longitud hocico-cloaca (LH), perímetro torácico (PT), longitud de cabeza (LC) y ancho de cabeza (AC). Las extracciones de sangre se practicaron a 10 animales de cada lote, 5 de cada sexo, por punción del seno post occipital. La alícuota de sangre extraída, se fraccionó en dos partes, una se trató con anticoagulante (EDTA) y la otra fue centrifugada para obtener suero. Estas muestras se preservaron refrigeradas $\left(5^{\circ} \mathrm{C}\right)$ hasta su procesamiento en el laboratorio.

Las pruebas hematológicas consistieron en la realización del hematocrito (por centrifugación de tubos 
Tabla 1. Efecto de las dietas sobre el peso vivo $(\mathrm{kg})$ y variables morfométricas $(\mathrm{cm})$.

\begin{tabular}{lcccccc}
\hline dieta & PV & LT & LH & PT & LC & AC \\
\hline A & $2,28 \mathrm{a} \pm 0,25$ & $81,59 \mathrm{ab} \pm 2,96$ & $41,41 \mathrm{a} \pm 1,24$ & $21,63 \mathrm{ab} \pm 1,03$ & $10,32 \mathrm{a} \pm 1,04$ & $6,48 \mathrm{a} \pm 0,43$ \\
B & $2,48 \mathrm{a} \pm 0,40$ & $84,51 \mathrm{a} \pm 6,66$ & $43,76 \mathrm{~b} \pm 3,16$ & $22,79 \mathrm{a} \pm 2,22$ & $10,49 \mathrm{a} \pm 0,73$ & $6,63 \mathrm{a} \pm 0,52$ \\
C & $2,02 \mathrm{~b} \pm 0,40$ & $79,81 \mathrm{~b} \pm 4,75$ & $41,41 \mathrm{a} \pm 2,95$ & $20,92 \mathrm{~b} \pm 1,72$ & $10,10 \mathrm{a} \pm 0,57$ & $6,41 \mathrm{a} \pm 0,44$ \\
ancova & 0,026 & 0,249 & 0,105 & 0,237 & 0,345 & 0,244 \\
p & $0,0004^{*}$ & $0,028^{*}$ & $0,014^{*}$ & $0,044^{*}$ & 0,306 & 0,331 \\
\hline
\end{tabular}

A: carne vacuna, B: pollo, C: pescado, PV: peso vivo, LT: longitud total, LH: longitud hocico-cloaca, PT: perímetro torácico, LC: longitud cabeza, AC: ancho cabeza. Las cifras indican media aritmética y desvío estándar. Ancova: análisis de covarianza (con el valor inicial de cada variable), p: significancia $(*<0,05)$. Letras distintas en cada columna expresan diferencias significativas (Tukey).

capilares a $12.000 \mathrm{rpm}$.) y la medición de hemoglobina, por espectrofotometría, técnica de la cianmetaHb, lecturas a $540 \mathrm{~nm}$, reactivos Wiener Lab, previa separación de los núcleos libres de los eritrocitos hemolizados, por centrifugación ${ }^{1}$.

Los parámetros bioquímicos se escogieron con el fin de que abarcaran la exploración del estado nutricional de los animales bajo ensayo. El proteinograma incluyó las determinaciones de proteínas totales por espectrofotometría, método del biuret, $540 \mathrm{~nm}$, reactivos Wiener, así como las fracciones seroproteicas (albúmina y globulinas alfa, beta y gamma), por electroforesis en soporte de acetato de celulosa, buffer de veronal sódico y coloración amidoschwartz, con ulterior transparentización y cuantificación por densitometría. La relación albúmina/globulinas (AG) se obtuvo por cálculo. El nitrógeno no proteico incluyó las determinaciones de urea y creatinina, obtenidas por espectrofotometría con reactivos Wiener. En el primer caso se empleó la técnica de la ureasa $(570 \mathrm{~nm})$ y en el segundo el método del picrato alcalino $(510 \mathrm{~nm})$. El glucograma incluyó las valoraciones de glucosa (técnica de la oxidasa/peroxidasa, lectura espectrofotométrica a $505 \mathrm{~nm}$, reactivos Wiener) y fructosamina (método de reducción del nitrotetrazolio, $530 \mathrm{~nm}$, reactivos Wiener). El lipidograma abarcó las mediciones de triglicéridos (glicerolfosfato-oxidasa/peroxidasa, $505 \mathrm{~nm}$, reactivos Wiener), colesterol total (colesterol-oxidasa-peroxidasa, $505 \mathrm{~nm}$, reactivos Wiener), colesterol ligado a lipoproteínas de alta y baja densidad, C-HDL y C-LDL (por precipitación selectiva de la lipoproteína y valoración enzimática del colesterol). Finalmente, el ionograma incluyó los dosajes de magnesio (método del xylidyl-blue, lecturas a $510 \mathrm{~nm}$ ), calcio (técnica de la cresolftaleíncomplexona, $570 \mathrm{~nm}$ ) y fósforo inorgánico (molibdato-ascorbato, $620 \mathrm{~nm}$, reactivos Wiener).

Bajo un diseño completamente aleatorizado, se calcularon las estadísticas descriptivas de tendencia central (media aritmética) y dispersión (desvío estándar), así como el análisis de la covarianza (ANCOVA), utilizando como covariables los valores iniciales de cada variable dependiente, a efectos de aumentar la potencia estadística mediante la reducción de la variabilidad. Para la comparación de medias se empleó el test de Tukey. Los cálculos se efectuaron con el auxilio del programa estadístico InfoStat Profesional (2008) ${ }^{5}$.

\section{RESULTADOS Y DISCUSIÓN}

La Tabla 1 indica que los ejemplares suplementados con pollo alcanzaron pesos más altos que los que consumieron carne vacuna y pescado. Además, los reptiles asignados al suplemento de origen aviar alcanzaron las mayores dimensiones corporales, señalando una mayor velocidad de crecimiento. Ninguna de las variables bajo estudio se modificó por el efecto sexo, circunstancia que ya había sido observada en otras experiencias realizadas con caimanes ${ }^{1}$. Sin embargo, otros autores hallaron que los neonatos machos de $C$. latirostris poseían cráneos más pequeños que las hembras, atribuyendo la diferencia al dimorfismo sexual ${ }^{13}$. En experiencias previas, caimanes que consumieron pollo también revelaron significativos incrementos del peso vivo y la longitud hocico-cloaca en relación a los que recibieron carne vacuna ${ }^{1}$.

Asimismo, de la Tabla 1 se desprende que el peso vivo fue la única variable dependiente relacionada a los parámetros morfométricos en que su covariable fue significativa $(p=0,026)$, circunstancia que se atribuye a la considerable variabilidad de pesos que mostraron los grupos al inicio de la experiencia, lo cual resalta las ventajas del ANCOVA al permitir desagregar tales diferencias ${ }^{5}$.

El consumo de pollo generó una ganancia de peso total promedio de $980 \mathrm{~g}$ y una ganancia de peso diaria promedio de $6 \mathrm{~g}$. Las dietas con carne de vaca y pescado mostraron ganancias totales de 880 y $550 \mathrm{~g}$ en cada caso, y ganancias diarias de 5 y $3 \mathrm{~g}$ respectivamente. Por otra parte, el crecimiento total fue de $11,04 \mathrm{~cm}$ y el crecimiento diario fue de $7 \mathrm{~mm}$ para el lote alimentado con pollo $(10,37 \mathrm{~cm}$ para carne de vaca y $9,18 \mathrm{~cm}$ para pescado, con crecimiento diario de 6 y $5 \mathrm{~mm}$ respectivamente). Las dietas ensayadas produjeron velocidades de crecimientos similares a las obtenidas por otros investigadores en Caiman crocodilus ${ }^{18}$ y Crocodylus acutus ${ }^{11}$.

En trabajos donde se compararon cuatro poblaciones de cocodrilos de los pantanos (Crocodylus morelet$t i$ ), dos de ellas estuvieron conformadas por individuos que nacieron en vida silvestre y otras dos por sujetos nacidos en cautiverio, observándose que los rasgos morfológicos estudiados fueron similares entre poblaciones y entre sexos. Las tasas de crecimiento corporal 
Tabla 2. Efecto de las dietas sobre las variables del proteinograma (g/dl).

\begin{tabular}{lccccccc}
\hline dieta & PR & AL & GT & GA & GB & GG & AG \\
\hline A & $4,65 \pm 0,25$ & $1,30 \pm 0,13$ & $2,44 \mathrm{a} \pm 0,22$ & $0,59 \mathrm{a} \pm 0,10$ & $1,08 \mathrm{a} \pm 0,07$ & $2,02 \mathrm{a} \pm 0,2$ & $0,39 \pm 0,07$ \\
B & $5,03 \pm 0,21$ & $1,34 \pm 0,20$ & $3,69 \mathrm{~b} \pm 0,30$ & $0,63 \mathrm{a} \pm 0,09$ & $0,88 \mathrm{~b} \pm 0,07$ & $1,84 \mathrm{~b} \pm 0,22$ & $0,37 \pm 0,08$ \\
C & $4,94 \pm 0,70$ & $1,29 \pm 0,43$ & $3,66 \mathrm{~b} \pm 0,48$ & $0,80 \mathrm{~b} \pm 0,17$ & $0,77 \mathrm{c} \pm 0,11$ & $2,09 \mathrm{a} \pm 0,37$ & $0,33 \pm 0,09$ \\
ancova & 0,529 & 0,168 & 0,514 & 0,637 & 0,990 & 0,145 & 0,888 \\
p & 0,275 & 0,983 & $0,0001^{*}$ & $0,005^{*}$ & $0,0003^{*}$ & $0,05^{*}$ & 0,322 \\
\hline
\end{tabular}

A: carne vacuna, B: pollo, C: pescado, PR: proteínas totales, AL: albúmina, GT: globulinas totales, GA: globulinas alfa, GB: globulinas beta, GG: globulinas gamma, AG: relación albúmina/globulinas. Las cifras indican media aritmética y desvío estándar. Ancova: análisis de covarianza (con el valor inicial de cada variable), p: significancia $(*<0,05)$. Letras distintas en cada columna expresan diferencias significativas (Tukey).

fueron significativamente diferentes entre poblaciones. Los individuos que nacieron en condiciones naturales crecieron más rápidamente que los nacidos en cautiverio ${ }^{19}$.

Otros investigadores aseveran que la carne blanca (pollo, pescado) es tan adecuada como la roja (vaca, cerdo) para la alimentación de los caimanes ${ }^{15}$. Los diseños de dietas para cocodrilos como $C$. acutus se basan principalmente en las sustituciones parciales de pescado por otros insumos, principalmente vísceras y diversos componentes de mamíferos domésticos o aves enteras ${ }^{10,11}$. Se ha registrado que el patrón de crecimiento corporal de cocodrilos sometidos a diferentes sistemas de producción, puede verse afectado por las condiciones del cautiverio, particularmente por los componentes y la frecuencia de la alimentación, la temperatura del agua y del aire, la densidad poblacional y otros factores ${ }^{10,16}$.

En otros ensayos se comprobó que una frecuencia de alimentación de cinco veces por semana (incluyendo carnes rojas), generó mayor ganancia de peso en ejemplares de C. acutus de un mes de edad. En el mismo trabajo se demostró que una menor frecuencia de alimentación (tres veces por semana), con una dieta que incluía harina de pescado, generó mayores aumentos de la longitud de los reptiles ${ }^{12}$. Neonatos de cocodrilos de pantano (C. moreletti) registraron significativas diferencias en su tasa de crecimiento y evolución del peso vivo al ser alimentados con diferentes dietas. La dieta que contenía sólo carne de pescado resultó ser menos eficiente con relación a la que incluyó pescado más vísceras bovinas y la basada en larvas de moscas más calcio ${ }^{10}$. Estos resultados se acercan a los alcanzados en nuestro trabajo, donde la carne de pescado reveló menor eficiencia para elevar el peso vivo y las demás variables morfométricas de $C$. yacare.

La Tabla 2 señala las modificaciones ocurridas en el proteinograma por efecto de las raciones suministradas. Se advierte que las proteínas totales, albúmina y relación albúmina/globulinas no fueron influenciadas significativamente por el tipo de suplemento. No obstante, surge que la suplementación con pollo produjo tasas más altas de albúmina, proteínas totales y globulinas totales que en los especimenes asignados a carne de vaca y pescado. Para dos especies del género Caiman otros investigadores obtuvieron valores disímiles de proteínas totales y globulinas, pues resultaron más altos en C. latirostris que en C. yacare $(5,76$ vs $5,61 \mathrm{~g} /$ dl y 3,07 vs $2,30 \mathrm{~g} / \mathrm{dl}$ respectivamente) ${ }^{20}$. La tasa de albúmina aquí registrada para los caimanes suplementados fue superior a la obtenida sobre la misma especie, tanto en cautiverio $(0,95 \mathrm{~g} / \mathrm{dl}$, con $\mathrm{AG}=0,29)$ como silvestres $(0,87 \mathrm{~g} / \mathrm{dl} \text { con } A G=0,23)^{21}$. Los mayores valores de albúmina registrados en nuestra experiencia quizás se relacionen con la calidad (digestibilidad) de las proteínas de la ración ${ }^{1}$.

Los animales que consumieron carne vacuna revelaron los más altos niveles séricos de urea $(0,17 \pm 0,06$ $\mathrm{g} / \mathrm{l}, \mathrm{p}=0,004)$ que los que recibieron pescado $(0,14$ $\pm 0,02 \mathrm{~g} / \mathrm{l})$ y pollo $(0,11 \pm 0,01 \mathrm{~g} / \mathrm{l})$. La creatininemia se mostró más elevada en los animales que consumieron pescado $(9,40 \pm 0,97 \mathrm{mg} / \mathrm{l}, \mathrm{p}=0,001)$, en relación a los que recibieron suplementos de carne bovina $(6,10 \pm 0,88$ $\mathrm{mg} / \mathrm{l})$ y pollo $(8,10 \pm 1,10 \mathrm{mg} / \mathrm{l})$. Para la especie bajo ensayo se consideran normales los guarismos de urea y creatinina cercanos a $0,11 \pm 0,02 \mathrm{~g} / \mathrm{l}$ y $5,25 \pm 1,06 \mathrm{mg} / 1$ respectivamente ${ }^{1}$.

Se citan significativas variaciones de urea sérica atribuibles al sexo, pues resultó mayor en hembras $(0,11$ $\mathrm{g} / \mathrm{l})$ que en machos $(0,09 \mathrm{~g} / \mathrm{l})$ y también por el tipo de alimentación, pues fue mayor en animales de criadero $(0,11 \mathrm{~g} / \mathrm{l})$ que en sujetos mantenidos en zoológicos $(0,09$ $\mathrm{g} / \mathrm{l})$. En cambio, para la creatinina no se encontraron diferencias significativas atribuibles al sexo $(5,54 \mathrm{mg} / \mathrm{l} \mathrm{en}$ hembras vs $5,05 \mathrm{mg} / 1$ en machos) ni a las condiciones de vida $(5,94 \mathrm{mg} / \mathrm{l}$ en criaderos y $5,61 \mathrm{mg} / \mathrm{l}$ en zoológicos) ${ }^{1}$. Además, en ensayos donde se incluyeron especimenes de C. latirostris y C. yacare, se observó que las concentraciones séricas de urea y creatinina se mostraron más elevadas en las estaciones cálidas que en las frías, quizás debido al incremento de la ingesta proteica y el consecuente aumento de las masas musculares ${ }^{1}$.

En el presente ensayo la glucemia se mostró más elevada $(p=0,141)$ en ejemplares suplementados con pescado $(1,57 \pm 0,37 \mathrm{~g} / \mathrm{l})$, con relación a los que consumieron carne y pollo $(1,16 \pm 0,15$ y $1,21 \pm 0,30 \mathrm{~g} / \mathrm{l})$. Consecuentemente, la fructosamina acompañó este comportamiento, presentándose significativamente incrementada $(p=0,004)$ en los animales que consumieron pescado ( $266 \pm 85,24 \mathrm{umol} / \mathrm{l})$, en relación a los que recibieron carne vacuna $(196 \pm 25,83$ umol/l) y pollo (178 $\pm 31,09 \mathrm{umol} / \mathrm{l})$. 
Tabla 3. Efecto de las dietas sobre las variables del lipidograma (g/l).

\begin{tabular}{lcccc}
\hline dieta & CT & TG & C-HDL & C-LDL \\
\hline A & $1,29 \mathrm{a} \pm 0,26$ & $1,40 \mathrm{a} \pm 1,01$ & $0,26 \mathrm{a} \pm 0,07$ & $0,39 \mathrm{a} \pm 0,08$ \\
$\mathrm{~B}$ & $1,59 \mathrm{~b} \pm 0,26$ & $0,68 \mathrm{~b} \pm 0,17$ & $0,33 \mathrm{~b} \pm 0,10$ & $0,67 \mathrm{~b} \pm 0,18$ \\
$\mathrm{C}$ & $1,50 \mathrm{~b} \pm 0,33$ & $0,29 \mathrm{~b} \pm 0,10$ & $0,52 \mathrm{~b} \pm 0,27$ & $0,34 \mathrm{a} \pm 0,17$ \\
ancova & 0,689 & 0,644 & 0,827 & 0,827 \\
$\mathrm{p}$ & $0,05^{*}$ & $0,019^{*}$ & $0,009^{*}$ & $0,04^{*}$ \\
\hline
\end{tabular}

A: carne vacuna, B: pollo, C: pescado, CT: colesterol total, TG: triglicéridos, C-HDL y C-LDL: colesterol ligado a lipoproteínas de alta y baja densidad. Las cifras indican media aritmética y desvío estándar. Ancova: análisis de covarianza (con el valor inicial de cada variable), p: significancia $(*<0,05)$. Letras distintas en cada columna expresan diferencias significativas (Tukey).

Tabla 4. Efecto de las dietas sobre las variables del ionograma.

\begin{tabular}{lccccc}
\hline dieta & $\begin{array}{c}\text { sodio } \\
(\mathrm{meq} / \mathrm{l})\end{array}$ & $\begin{array}{c}\text { potasio } \\
(\mathrm{meq} / \mathrm{l})\end{array}$ & $\begin{array}{c}\text { calcio } \\
(\mathrm{mg} / \mathrm{dl})\end{array}$ & $\begin{array}{c}\text { fósforo } \\
(\mathrm{mg} / \mathrm{dl})\end{array}$ & $\begin{array}{c}\text { magnesio } \\
(\mathrm{mg} / \mathrm{dl})\end{array}$ \\
\hline $\mathrm{A}$ & $141 \mathrm{a} \pm 2,11$ & $5,37 \pm 0,46$ & $10,44 \mathrm{a} \pm 0,38$ & $4,09 \mathrm{a} \pm 0,40$ & $2,77 \mathrm{a} \pm 0,22$ \\
$\mathrm{~B}$ & $147 \mathrm{a} \pm 6,26$ & $5,20 \pm 0,30$ & $9,37 \mathrm{~b} \pm 0,61$ & $5,03 \mathrm{~b} \pm 1,04$ & $2,55 \mathrm{a} \pm 0,49$ \\
$\mathrm{C}$ & $129 \mathrm{~b} \pm 5,52$ & $5,17 \pm 0,67$ & $11,05 \mathrm{a} \pm 0,64$ & $3,07 \mathrm{c} \pm 0,94$ & $2,34 \mathrm{~b} \pm 0,47$ \\
ancova & 0,156 & 0,217 & 0,601 & 0,532 & 0,586 \\
p & $0,05^{*}$ & $0,826^{*}$ & $0,0003^{*}$ & $0,014^{*}$ & $0,05^{*}$ \\
\hline
\end{tabular}

A: carne vacuna, B: pollo, C: pescado. Las cifras indican media aritmética y desvío estándar. Ancova: análisis de covarianza (con el valor inicial de cada variable), p: significancia $(*<0,05)$. Letras distintas en cada columna expresan diferencias significativas (Tukey).

En ensayos realizados sobre $C$. moreletti se hallaron glucemias más elevadas en ejemplares de vida silvestre que en animales mantenidos en cautiverio ${ }^{9}$. La glucosa sérica también varió según las condiciones del hábitat, habiéndose encontrado diferencias significativas entre las glucemias de caimanes mantenidos en criaderos y en zoológicos ${ }^{1}$.

En la Tabla 3 puede observarse el comportamiento de las variables incluidas en el lipidograma, de acuerdo a los tres suplementos suministrados en la experiencia. El colesterol total resultó más alto en especimenes que consumieron pollo y pescado $(1,59$ y $1,50 \mathrm{~g} / 1$ respectivamente), con relación a los que recibieron carne de vaca $(1,29 \mathrm{~g} / \mathrm{l}, \mathrm{p}=0,05)$. Los triglicéridos fueron significativamente más altos en la dieta cárnea $(1,40$ $\mathrm{g} / \mathrm{l})$ que en las complementadas con pollo y pescado ( 0,68 y $0,29 \mathrm{~g} / 1$ respectivamente). Existen referencias señalando que el género Caiman respondería al patrón metabólico lipoproteico tipo "LDL" (donde C-LDL > C-HDL) ${ }^{1}$. En la presente experiencia, esta aseveración fue válida en los animales que consumieron carne vacuna y pollo, no así en los reptiles suplementados con pescado, donde los niveles de C-HDL fueron significativamente mayores $(\mathrm{p}=0,009)$.

En C. moreletti se hallaron tenores de colesterol total más elevados en hembras $(2,80 \mathrm{~g} / \mathrm{l})$ que en machos $(2,40 \mathrm{~g} / \mathrm{l})$, señalándose concentraciones séricas más altas en ejemplares en cautiverio $(2,80 \mathrm{~g} / \mathrm{l})$ que en aquéllos de vida silvestre $(2,18 \mathrm{~g} / \mathrm{l})$, atribuyéndose dicha variación a la dieta y a la frecuencia de alimentación ${ }^{9}$.
Con respecto al hemograma, los animales que consumieron pescado manifestaron elevaciones (no significativas) de hematocrito (24 $\pm 1,60 \%)$ y hemoglobina $(6,91 \pm 0,71$ $\mathrm{g} / \mathrm{dl}$ ) en relación a los asignados a la carne vacuna $(23 \pm 1,25 \%$ y $6,81 \mathrm{~g} /$ dl) y pollo $(22 \pm 1,90 \%$ y $6,41 \pm 0,86$ $\mathrm{g} / \mathrm{dl}$ ). El rango de valores hallados en los hemogramas del presente ensayo se aproximan a los referenciados para ejemplares sub-adultos de $C$. latirostris y $C$. yacare, que fueron de $21,2 \pm 3,5 \%$ para hematocrito y de $6,12 \pm 1,08 \mathrm{~g} / \mathrm{dl}$ para hemoglobina ${ }^{1}$. En dicha publicación se hace referencia a la ausencia de diferencias atribuibles al sexo de los animales, en concordancia a lo observado en nuestra experiencia. Los parámetros de la serie roja están reputados como buenos indicadores del estado nutricional de los caimanes, ya que una alimentación inapropiada es capaz de generar marcados descensos de hemoglobina y hematocrito (hasta niveles de $17 \%)^{4}$.

En la Tabla 4 se consignan las variaciones del ionograma de C. yacare debidas a la incorporación de distintos suplementos a la dieta base. Algunos valores de electrolitos séricos analizados en nuestro trabajo, son similares a los citados para la misma especie por otros investigadores (sodio 148,2 meq/1; potasio 4,58 meq/1 y fósforo inorgánico $5,16 \mathrm{mg} / \mathrm{dl}$ ) ${ }^{21}$. Se afirma que la magnesemia es significativamente más alta en machos $(2,71$ $\mathrm{mg} / \mathrm{dl})$ que en hembras $(2,52 \mathrm{mg} / \mathrm{dl})$; asimismo que la calcemia y la fostatemia disminuyen significativamente en función del aumento de la edad de los caimanes ${ }^{1}$.

Los niveles de algunos electrolitos séricos disminuirían significativamente durante el invierno, posiblemente debido a la disminución del ingreso de minerales causado por la interrupción de la alimentación durante la estación fría ${ }^{6}$. Por otra parte, la inadecuada relación $\mathrm{Ca}: \mathrm{P}$ en la dieta de los reptiles puede conducir a trastornos óseos y dentales. Así, en siete ejemplares de caimanes del Orinoco (Crocodylus intermedius) se detectó ausencia parcial o total de los dientes, lo cual reveló la presencia de un marcado hiperparatiroidismo secundario nutricional ${ }^{2}$.

En conclusión, surge que los caimanes que recibieron la ración suplementada con pollo revelaron significativos incrementos de peso vivo, longitud total, longitud hocico-cloaca, perímetro torácico, fósforo inorgánico, sodio, globulinas totales, globulinas beta, colesterol total y C-LDL. Los reptiles suplementados con carne vacuna mostraron mayores niveles de urea, triglicéridos y magnesio. Los animales que consumieron 
pescado exhibieron mayores tasas sanguíneas de calcio, creatinina, fructosamina, C-HDL y globulinas alfa y gamma. En ningún grupo se registraron casos compatibles con deficiencias nutricionales, infiriéndose que los tres suplementos utilizados revelaron ser útiles para la alimentación de C. yacare, sobresaliendo el de origen aviar por su mayor impacto sobre los indicadores bioquímicos, el peso y las dimensiones corporales de los caimanes.

\section{REFERENCIAS}

1. Barboza NN, Mussart NB, Koza GA, Coppo JA. 2002. Internal environment in juvenile specimens of Caiman latirostris and Caiman yacare from Argentina. Physiological variations according to species, sex, liveweight, size, and season of the year. Rev Electron Veter 13: 1-23.

2. Boede EO, Sogbez E. 2000. Enfermedades en caimanes del Orinoco (Crocodylus intermedius) y caimanes de la costa (Crocodylus acutus) mantenidos en zoocriaderos venezolanos. Rev Cient FCV-LUZ, 10: 328-338.

3. Borteiro C, Prigioni C, García JE, Tedros M, Gutiérrez F, Kolenc F. 2006. Geographic distribution and conservation status of Caiman latirostris (Crocodylia, Alligatoridae) in Uruguay. Phyllomedusa 5: 97-108.

4. Ferreyra H, Uhart M. 2001. Evaluación y evolución del estado sanitario de Caiman latirostris y Caiman yacare en el Refugio El Cachapé. Boletín Técnico de la Fundación Vida Silvestre Argentina 55: 1-15.

5. InfoStat. 2008. Grupo InfoStat FCA-Córdoba, Argentina.

6. Lane TJ. 1996. Crocodilians. In: Reptile Medicine and Surgery (Mader DR ed.), Saunders, Philadelphia, p. 78-98.

7. Larriera A, Imhof A. 2006. Proyecto yacaré: cosecha de huevos para cría en granjas del género Caiman en Argentina. In: Manejo de fauna silvestre en Argentina (Bolkovic ML, Ramadori D, ed.), Publ. Secretaría de Ambiente y Desarrollo Sustentable, Buenos Aires, p. 51-64.

8. Larriera A, Piña C, Dacey T. 2008. Conservación, manejo y uso sustentable de los cocodrilos en Cuba. Anales II Taller Nacional "Situación actual del cocodrilo cubano Crocodylus rhombifer”, Ciénaga de Zapata, Cuba.

9. Padilla SE, Weber M, Jacobson E. 2009. Comparación de anticoagulantes de heparina de litio y sodio en la bioquímica plasmática del cocodrilo de pantano (Crocodylus moreletti) en Campeche, México. Vet Méx 40: 203-211.

10. Pérez-Gómez M, Naranjo C, Reyes B, Vega I. 2009. Influencia de dos tipos de dietas sobre la talla y el peso corporal en neonatos de Crocodylus acutus Cuvier 1807 del zoocriadero de Manzanillo, Cuba. Acta Zool Mex 25: 151-160.
11. Pérez O, Escobedo-Galván A. 2007. Crecimiento en cautiverio de Crocodylus acutus (Cuvier, 1807) en Tumbes, Perú. Rev Peruana Biol 14: 221-223.

12. Piedra CL, Bolaños JR, Sánchez JR. 1996. Evaluación del crecimiento de neonatos de Crocodylus acutus (Crocodilia: Crocodylidae) en cautiverio. Rev Biol Trop 44: 289-293.

13. Piña C, Larriera A. 2002. Caiman latirostris growth: the effect of a management technique on the supplied temperature. Aquaculture 211: 387-392.

14. Piña C, Larriera A, Siroski P, Verdade LM. 2007. Cranial sexual discrimination in hatchling broad-snouted caiman (Caiman latirostris). Iheringia. Série Zoologia (Porto Alegre) 97: 17-20.

15. Pinheiro MS, Lavorenti A. 2001. Growth of broad-nosed caiman, Caiman latirostris (Daudin, 1802) hatchlings, fed with diets of animal origin. Braz J Biol 61: 421-429.

16. Poletta GL, Larriera A, Siroski PA. 2008. Broad snouted caiman (Caiman latirostris) growth under different rearing densities. Aquaculture 280: 264-266.

17. Prado W, Gómez O, Balla P. 2001. Crianza en cautiverio y reintroducción de individuos. Boletín Técnico de la Fundación Vida Silvestre Argentina 55: 26-38.

18. Rodríguez MA, Clavijo LA, López FO, Gerardino A, Ceballos FC, Arboleda JJ, Silva AE, Guerrero PH. 1996, Avances en la nutrición de Caiman crocodilus. Proceedings of Working Meeting of the Crocodile Specialist Group (Santa Fe, Argentina), p. 347-354.

19. Serna R, Zúñiga J, Díaz P, Clemente F, Pérez A, Reta GL. 2010. Variabilidad morfológica y crecimiento corporal de cuatro poblaciones de Crocodylus moreletii en cautiverio. Rev Mex Biodiv 81: 713-719.

20. Troiano JC, Althaus RL, Malinskas G. 1997. Perfil bioquímico sanguíneo de las especies del género Caiman en condiciones de cautividad. Rev Españ Herpetol 11: 31-34.

21. Uhart M, Prado W, Beldoménico P, Rossetti C, Ferryera MC, Martinez A, Bardón JC, Avilés G, Karesh W. 2001. Estudios sanitarios comparativos de yacarés (Caiman latirostris y Caiman yacare) silvestres y cautivos. Boletín Técnico Fundación Vida Silvestre Argentina 55: 1-15.

22. Verdade LM, Santiago ME. 1991. Studybook regional do jacaré-do-papo-amarelo (Caiman latirostris), Ed. Esalq/ USP, Piracicaba (Brasil), $34 \mathrm{p}$.

23. Waller T, Minucci PA. 1993. Relevamiento de la distribución, hábitat y abundancia de los crocodilios de la Provincia de Corrientes, Argentina. Memorias 1ra. Reunión Regional del Grupo de Especialistas en Cocodrilos, Santa Marta (Colombia), p. 341-385. 